\title{
IDENTIFICACIÓN DEL DOCUMENTO DE ACUERDO AL CÓDIGO DEL CUADRO DE CLASIFICACIÓN DOCUMENTAL: CASO UNIVERSIDAD POLITECNICA SALESIANA DEL ECUADOR'
}

\author{
Willian Fernando Ladino-Jiménez ${ }^{2}$ \\ María José Llanos Campoverde 3
}

RESUMEN

Ante la falta de una cultura archivística en una organización, los documentos realizan una tramitación interna sin tener un control o conocer a qué proceso pertenece, de igual manera la terminología se desconoce confundiendo "conservar" como incorporar los documentos en una carpeta, folder, sobre y ubicarlos en algún espacio físico a la espera del personal que trabaja en un Archivo. La Universidad Politécnica Salesiana en su ambiente organizacional apuesta para que el documento desde su creación tenga consignado una identificación de acuerdo al código del cuadro de clasificación documental. El código estará reflejado en todo el proceso administrativo y académico con la compañía de una estructura alfanumérica que permita agruparlos bajo un mismo número de trámite o asunto. El incorporar el código y la estructura alfanumérica permitirá que el personal ordene la documentación de acuerdo a la estructura dada en el Cuadro de Clasificación Documental.

Palabras clave: Cuadro de clasificación. Tipos Documentales. Archivo. Trámite. Código de Clasificación. Gestión Documental.

\footnotetext{
${ }^{1}$ Trabalho do Eixo Temático "Os sistemas de gestão de documentos e a gestão da qualidade" apresentado em 30 de agosto de 2017 no VIII Congresso Ibero-Americano de Arquivos Universitários (CIAU).

${ }^{2}$ Secretário Técnico de Arquivo e Documentação da Universidad Politecnica Salesiana (UPS), Equador. E-mail: wladino@ups.edu.ec.

${ }^{3}$ Licenciada em Ciências de Comunicação Social - Publicidade e Relações Públicas pela Universidad de Cuenca, Equador.
} 
Willian Fernando Ladino-Jiménez e María José Llanos Campoverde

\section{LA NECESIDAD DEL ARCHIVO INSTITUCIONAL DE LA UPS}

a Universidad Politécnica Salesiana (UPS) como institución de enseñanza superior, fue
creada en el año de 1994 con su matriz en Cuenca, sus sedes en la ciudad de Guayaquil
y Quito. En los 22 años de vida institucional, se ha dado la necesidad de crear un sistema que permita administrar la documentación generada tanto en un ambiente académico, administrativo, investigativo, cultural y de vinculación con la sociedad. De igual manera la necesidad de conservar documentación relativa a la historia - todavía breve - de la propia institución.

Ante la falta de una cultura archivística al interior de la organización se ha entendido por el término "conservar" el incorporar los documentos en una carpeta, folder, sobre, etc. Y estos dentro de una caja donde serán ubicados en el rincón de alguna zona de la oficina.

A finales del año 2010, con resolución del Consejo Superior, se aprueba la creación de la Secretaría Técnica de Archivo y Documentación con la finalidad de diseñar, implementar un sistema de gestión documental.

Desde entonces, la UPS ha entendido que "conservar" significa no solo "no destruir", ni "guardar los papeles", sino hacerlo de forma que "los papeles" por su naturaleza se consideren documentos que contienen información y constituye un activo fijo patrimonial que se ha de preservar para el presente y futuro (Millán, 2016), mismos que - según el Cuadro de Clasificación aprobado por la universidad - se agrupan de acuerdo a las funciones y actividades, creando así los expedientes simples o compuestos de elementos homogéneos, tanto en su objeto como en su estructura de tipos documentales Rodríguez (2010) "siendo el corazón no solo del proceso de clasificación sino de la gestión integral de los sistemas electrónicos de gestión de documentos", documentos éstos identificados, controlados, localizados, accesibles, legibles, con las firmas y aquellas características que les confieran identidad, autenticidad.

Informação Arquivística, Rio de Janeiro, v.6, n. 1, p. 105-121, jan./jun., 2017 
Willian Fernando Ladino-Jiménez e María José Llanos Campoverde

La universidad cuenta con un sistema de archivos estructurados y definidos a nivel nacional, proyectándose al diseño e implementación de un sistema de gestión documental.

\begin{tabular}{|c|c|}
\hline Sistema de Archivos & Sistema de Gestión Documental \\
\hline Archivo Institucional o Histórico & Gestión por procesos \\
\hline Archivo Definitivo & e-administración \\
\hline Archivo Intermedio & Control y calidad de datos \\
\hline Archivo de Gestión & \\
\hline
\end{tabular}

La UPS ha entendido también que un Archivo, sea de documentos físicos o electrónicos, no es un depósito muerto, es un instrumento eficaz y eficiente que gestiona el conjunto de documentos generados y recibidos por la organización en el desempeño de sus funciones Mundet, J. R. C. (2001). Un archivo es un instrumento vivo que necesita ser revisado permanente y sistemáticamente, no solo con el fin de garantizar tanto la existencia como el estado de conservación de los bienes depositados, sino también, para ir expurgando aquellos documentos que, en función del tipo documental al que pertenecen, a los tiempos de vida asignados de acuerdo al ciclo de vida como señala (de la Nación, A. G, 1996) donde se realiza el “control y seguimiento desde la producción o recepción, el trámite, la distribución, la consulta, la retención, el almacenamiento, la recuperación, la preservación su disposición de conservación definitiva" o han superado su periodo de vigencia y deben ser eliminados o con el uso de tecnologías ser desmaterializados para pasar de un formato "papel" a un formato electrónico, más compacto y con mejores condiciones de conservación, acceso y seguridad física.

La Universidad - además del Rectorado - cuenta con tres sedes en Cuenca, Quito y Guayaquil, y varias extensiones geográficamente dispersas aunque asociadas a las sedes, por 
Willian Fernando Ladino-Jiménez e María José Llanos Campoverde

lo tanto al momento del paso de los documentos a otras instancias técnicas, se pierde "el poder" sobre los mismos, obliga a que los archivos respondan a estructuras, tipos documentales, criterios, normas, procedimientos e incluso codificaciones, parámetros y sistemas de gestión únicos y comunes e impone también un Sistema de Archivos que, al menos en lo académico se estructura en tres niveles:

- Nivel de Gestión: Que afecta a los trámites en curso, expedientes en proceso o que, incluso habiendo finalizado, deben permanecer durante un cierto número de años bajo la competencia y en el archivo físico de la unidad administrativa o académica gestora.

- Nivel Intermedio a nivel de Sede: Que afecta a los trámites finalizados y a los expedientes cerrados para los que, habiendo transcurrido los plazos de permanencia en el archivo de la unidad administrativa o académica gestora, no han cumplido "los plazos de vida" de los tipos documentales implicados.

- Nivel Definitivo a nivel de Sede: Los expedientes son relevantes para su propia Sede. Tienen un plazo precaucional indefinido debido a procesos de acreditación y evaluación de la universidad ante organismo externos o porque no se tiene un cálculo definitivo hasta que edad una persona puede continuar con sus estudios de posgrado.

- Nivel Histórico: Que incluye los documentos que tienen la calificación de "históricos" porque su valor es independiente del tiempo, o que, no teniendo esa calificación, se ha decidido por los órganos competentes, con la aprobación de las autoridades de la universidad, pasen temporal o indefinidamente a este nivel de Archivo.

\section{EL SISTEMA DE GESTION DEL ARCHIVO INSTITUCIONAL}

En el punto anterior se hizo mención que un Archivo, o un Sistema de Archivos como el 
Willian Fernando Ladino-Jiménez e María José Llanos Campoverde

de la UPS, estructurado en niveles y geográficamente dispersos, requiere inevitablemente de un Sistema Informático de Gestión. Sistema éste basado no solo en una Base de Datos (BD) de referencias tanto de personas, de títulos, de expedientes o de los diferentes tipos documentales, sino también de una BD de referencias de ubicaciones y BD de imágenes de documentos. Sin tomar en consideración, por el momento, la gestión de elementos documentales constituidos por documentos sonoros, películas, etc.

Digamos en primer lugar que, con independencia de determinar los objetivos del sistema y desarrollar las funcionalidades de estos sistemas, estas BD deben cumplir una serie de criterios que - por ejemplo para la BD de referencias de Expedientes de Titulados - serían, como mínimo los de:

- Integridad: los expedientes de los títulos que deben figurar en la BD de cada nivel de Gestión e Intermedios de Sede figuren en la BD y que no pueden existir duplicados ni de los titulados, ni de los títulos de un titulado.

- Identificación: un mismo titulado tenga una única referencia en cada nivel de archivo, y que una referencia no este asignada más que a un único titulado.

- Calidad: la descripción de un titulado, dado que se hace normalmente a través de sus apellidos y nombre y su género, la grafía de los apellidos debe estar "normalizada".

- Congruencia externa: como las informaciones de la BD de los Archivos Intermedios de Sede provienen habitualmente de diferentes orígenes, incluso externos a la propia Institución, es normal encontrar diferencias entre informaciones que debieran ser la misma, o falta de congruencia entre informaciones entre las que existe una relación lógica.

- Congruencia interna: la falta de conformidad entre informaciones se da para informaciones de la propia Institución.

A fin de comprender mejor el tema planteado es de gran importancia hablar a título de ejemplo, de la falta de integridad de las "entidades" titulados y títulos, o de las referencias documentales, como pueden ser las de los expedientes académicos de titulados. 
Willian Fernando Ladino-Jiménez e María José Llanos Campoverde

Para crear la BD de titulados y títulos, y tomando en cuenta que no existía en la universidad un censo oficial de egresados mínimamente fiable, sino libros de Excel parciales, no contrastadas, integradas ni verificadas contra los archivos de expedientes académicos que - por otra parte - no respondían a una estructura ni ordenación común y única, se pensó en recurrir a una metodología clásica de cargar y consolidar sobre una Excel todas las entidadespersona, y todos los datos de cada entidad persona que se encontró en las diferentes fuentes de acceso, contrastar estas informaciones contra los expedientes que se localizaron, contra lo que existía en el registro de títulos de la Secretaría Nacional de Educación Superior Ciencia y Tecnología (SENESCYT) del Ecuador, y consolidar sobre el documento Excel las nuevas informaciones y nuevos titulados encontrados, además se contrastó con una lista de certificados emitidos por la universidad a demanda de la SENESCYT, y trabajar con diferentes filtros para detectar fallos como paso previo a la generación de esta BD del sistema y para crear censos por promociones con la finalidad - no solo de disponer de una BD adecuada y verificada, sino también la de crear un archivo físico de expedientes académicos ordenado por promociones y dentro de cada promoción en alfabético de apellidos y nombre.

Pensar que la referencia que sirve de identificador de un expediente académico, no puede ser la de los apellidos y nombres, y no sólo porque es una referencia muy larga, con gran número de caracteres y grandes posibilidades de cometer errores, sino porque, superadas estas dificultades, nos encontraríamos con titulados diferentes pero homónimos, para cuya discriminación habría que añadir al identificador otro dato personal e invariable, como podría ser la fecha de nacimiento.

Es por ello que, se tomó como referencia de identificación de la persona el número de cédula, el cual incluye habitualmente un carácter de control, que se calcula mediante un algoritmo público y que garantiza que el identificador es correcto. Pero se debe tener presente que los sistemas informáticos utilizados en la gestión académica no siempre han incluido esta verificación en sus programas, lo que unido a otras carencias funcionales permite que:

Informação Arquivística, Rio de Janeiro, v.6, n. 1, p. 105-121, jan./jun., 2017 
Willian Fernando Ladino-Jiménez e María José Llanos Campoverde

- Existan personas con el número de cédula erróneo, que serán de difícil o imposible identificación y localización en el sistema:

$>0102366887$ y 0102366587 (Erróneo)

CABRERA MENESES Pedro Guillermo

$>0102552718$ y 1002552718 (Erróneo)

CARRION ASTUDILLO Cumanda Marlene

- Existan dos personas con apellidos y nombres completamente diferentes con el mismo número de Cédula:

> 1713461091 CABASCANGO CARRILLO Sonia del Pilar

VELEZ CARPIO Magdalena Hortencia

0200063832 GARCIA SALTOS Ruth Alicia

LUCIO GAYBOR Pedro Pablo

- Existan dos personas con apellidos y nombres con pequeñas diferencias de grafía y con el mismo número de Cédula, sin que pueda afirmarse si se trata de dos personas diferentes o de dos "clones" de la misma persona:

$>1101046645$ VELEZ PALACIO Nivea Luz Maria

VELEZ PALACIO Nivea Luz Maria Augusta

1707657688 VILLACRECES PAREDES Filomena el Pilar

VILLACRESES PAREDES Filomena el Pilar

- Existan dos personas con idéntica grafía de apellidos y nombres con diferentes números de cédula, correctos además desde el punto de vista del cálculo del dígito de control, sin que pueda afirmarse si es la misma o dos homónimos:

$>1708470073$ y 1102132345 QUEZADA OCHOA Angel Rodrigo

$>1303514200$ y 1706878491 SALTOS COLOMA Shelly Fabian

Informação Arquivística, Rio de Janeiro, v.6, n. 1, p. 105-121, jan./jun., 2017 
$>7515275,007515275,1003464441$ y 9800366327 GONZALEZ PORTELA Maria Lujan

- Existan personas con apellidos y nombres con pequeñas diferencias formales (posibles clones), con diferentes números de cédula, sin que pueda afirmarse si es la misma persona o dos diferentes casi "homónimas":

> 0906214880 ORELLANA JIMENEZ Sonnia Basilia

> 0906241880 ORELLANA JIMENEZ Sonnia Basilisa

$>0908391675$ PINCAY JACOME Enma

> 0907781314 PINCAY JACOME Emma del Rocio

- Existan números de cédula a la cual no corresponde a ningún ciudadano debido a la digitación errónea de un número:

> 1716701436 CALUNGA SALAS Javier

$>1935276$ DELPORTE, Jozef

> 0915016802 JIMENEZ CARRASCO Manuel (Mauricio Gabriel)

- Existan personas que por su estado migratorio cuentan con dos números de identificación como *EXT (otorgado por el Registro Civil a extranjeros residentes) y número de cédula perteneciente a su país de origen:

$>86079199$ y 0106429608

$>1721017620$ y 9958 pas GARFIAS ALVAREZ Filomena

- Pero, lo mismo podemos afirmar en cuanto a las denominaciones de los Títulos. ¿Debe un título llevar asignación de género?

$>1715425219$ CANDO CARRERA Luis Eduardo

LICENCIADA EN COMUNICACION SOCIAL CON ESPECIALIDAD EDUCATIVA

Informação Arquivística, Rio de Janeiro, v.6, n. 1, p. 105-121, jan./jun., 2017 
Willian Fernando Ladino-Jiménez e María José Llanos Campoverde

\section{BAQUERO OSORIO Renato Tulio}

\section{LICENCIADO (A) EN COMUNICACION SOCIAL}

Con los casos anteriores, podemos ver que implementar un sistema de gestión de Archivos, como señala, Sánchez Gómez, A. E. (2007), permite:

- Tratar los documentos de archivo, bajo lineamientos e instrumentos técnicos normalizados.

- Normalizar los diferentes tipos documentales que se producen.

- Unificar criterios para la organización (clasificación, ordenación y descripción) de los documentos de todas las dependencias.

- Definir y aplicar lineamientos para la selección y eliminación de documentos, y la preservación permanente de aquellos considerados como históricos.

- Aplicar estamentos legales nacionales e institucionales en materia de archivo, así como establecer normas para la correcta gestión de los documentos.

- Coordinar la simplificación, normalización y difusión de los procesos administrativos.

- Diseñar sistemas de información, bases de datos y páginas web que faciliten la labor administrativa, el registro y control de entrada, salida y trámite, así como la descripción de los documentos de archivo.

- Establecer planes, programas y proyectos para la desinfección, conservación y preservación de los documentos y los espacios físicos de las unidades de archivo.

- Velar por la aplicación de políticas de seguridad industrial a fin de garantizar el buen estado de salud del recurso humano que maneja, organiza y manipula documentos de archivo.

\section{EL SISTEMA DE ARCHIVOS}

Con el objeto de establecer lineamentos técnicos normalizados, unificar criterios para la 
Willian Fernando Ladino-Jiménez e María José Llanos Campoverde

organización, establecer estamentos legales nacionales, diseñar sistemas de información, bases de datos, programas y proyectos de desinfección y permitir el acceso a la documentación. La UPS institucionaliza a través de su Carta de Navegación, como objetivo estratégico "5.9. La comunidad universitaria de la UPS cuenta con información gestionada y conservada eficientemente".

\subsection{El Archivo Histórico}

La Universidad Politécnica Salesiana al ser joven y ser un proyecto que recién viene encaminado desde el año 2010, se le considera como Histórico aun en un estado de Archivo Institucional, a pesar de contener tipos documentales que por su naturaleza son históricos.

Este Archivo tal como fue concebido en la UPS, se encarga de analizar, proponer mejoras en la conservación y organización de los documentos sin importar su soporte y llevar a cabo los trabajos de tratamiento archivístico y conservación que correspondan con el tipo documental de cada uno para finalmente poder poner los documentos a disposición de las autoridades de la universidad, instituciones externas e investigadores interesados.

\subsection{El Archivo Intermedio}

Custodia la documentación que de acuerdo a su ciclo vital ha perdido vigencia administrativa y debe tener un plazo precaucional. Los documentos son transferidos desde las oficinas de gestión a otro espacio con las condiciones óptimas de temperatura, humedad y sanidad para que la documentación siga integra e intacta en el tiempo.

Informação Arquivística, Rio de Janeiro, v.6, n. 1, p. 105-121, jan./jun., 2017 
Willian Fernando Ladino-Jiménez e María José Llanos Campoverde

\section{EI CUADRO DE CLASIFICACIÓN DE TIPOS DOCUMENTALES DESDE LA CREACIÓN DE UN DOCUMENTO}

Definir cuál es el modelo de clasificación adecuado para implementarse en una organización dependerá de varios aspectos de acuerdo a la realidad estructural y funcional en la que se encuentre. Según (Heredia Herrera, 2010) Durante algún tiempo optamos por el cuadro de clasificación orgánico, admisible cuando la estructura de las Organizaciones no sufría variaciones pero pronto los continuos cambios institucionales lo hicieron difícil de mantener. Se recurrió a las funciones como más estables para configurar los parámetros del cuadro de clasificación orgánico-funcional, más funcional que orgánico que tuvo sus dificultades para plasmarlo en un formato único y plano. En esta ocasión basados en los 4 pilares de la UPS: docencia, investigación, Vinculación y administrativa implementa el cuadro de clasificación funcional.

Como explica Sánchez Gómez, A. E. (2015), el Cuadro de Clasificación:

- Proporciona vínculos entre documentos simples que se acumulan para proporcionar memoria continuada de la actividad.

- Asegura que los documentos sean nombrados de una forma consistente en el tiempo.

- Ayuda en la recuperación de todos los documentos relativos a una función o actividad particular.

- Determina seguridad en la protección y acceso apropiado a los documentos por categorías

- Asigna permisos de usuario para acceder a, o actuar sobre, grupos particulares de documentos.

- Distribuye responsabilidades para la gestión de determinadas categorías de documentos.

- Distribuye documentos para trabajar con ellos.

- Determina períodos de retención y acciones de selección adecuados para los 


\section{Willian Fernando Ladino-Jiménez e María José Llanos Campoverde}

documentos.

En ocasiones pensamos que la mejor manera es adoptar un modelo ya construido por otras organizaciones, sin darnos cuenta, que se convertirá en una desventaja en el momento de la aplicación.

- Falta de conocimiento de la organización y de ajuste adecuado con las necesidades de todo tipo específicas de nuestra institución.

- Falta de conocimiento de la producción de tipos documentales a considerar y de las necesidades de parametrización de los mismos.

- Falta del necesario análisis, aprendizaje, formación y sentido crítico de quienes trabajan en el Archivo en cuanto a "los por qué" de lo que hacen.

La UPS ve al CCD como aquel proceso de sinapsis, en donde un tipo documental que no se encuentre relacionado con una actividad específica y a la vez con una función, imposibilitará o dificultará en gran medida el proceso de búsqueda o acceso de información, encontrar o conocer lo qué sucedió con el trámite completo, cuáles son los tiempos de conservación de los documentos de este tipo, y cuáles son los plazos de revisión y de realización de los trabajos archivísticos que garanticen su adecuada conservación.

En el caso concreto de las universidades, el autor Michel ROBERGE, ha llegado a establecer un modelo que ha sido asumido por algunas universidades, y que ha sido publicado bajo el título "Lo esencial de la gestión documental" ISBN 978-2-9811275-2-5. A fin de tener una base de partida, y tras consultas efectuadas en un conjunto amplio de universidades en Ecuador, se optó asumir ese "modelo" como la base de partida del Cuadro de Clasificación Documental de la UPS, y adecuarlo a las actividades concretas de la universidad, tomando en consideración los comentarios, propuestas y recomendaciones que se reciban de los usuarios. EI CCD se estructura, en 13 categorías, que corresponden a las funciones básicas de la UPS. Cada una de estas categorías se estructura en "Clases", éstas a su vez en "Subclases", y cada Subclase en "Divisiones", Subdivisiones y - finalmente - estas identifican el Tipo Documental.

Para uno de los tipos documentales además de contener lo anterior, se establece un 
Willian Fernando Ladino-Jiménez e María José Llanos Campoverde

tiempo de retención en los diferentes niveles de Archivo, cada uno se encuentra acompañado con una descripción de base legal, donde se cita: la ley, reglamento, normativa, política, instructivo, que permita referenciar el tiempo de retención de cada Tipo Documental.

Así tenemos, por ejemplo:

$>$ Categoría: GESTION DE LOS RECURSOS ECONOMICOS Y FINANCIEROS

$>\quad$ Clase: PRESUPUESTO DE LA UNIVERSIDAD POLITÉCNICA SALESIANA

$>\quad$ Subclase: EJECUCIÓN DEL PRESUPUESTO UNIVERSITARIO

$>$ División: PAGO A PROVEEDORES

$>\quad$ Subdivisión: PAGO POR MANTENIMIENTO DE HARDWARE

$>\quad$ Tipo documental

- Solicitud para mantenimiento de hardware;

- Proforma para mantenimiento de hardware;

- Notificación de aprobación / no aprobación de pago por mantenimiento de hardware;

- Informe técnico sobre el mantenimiento de hardware;

- Solicitud de pago por mantenimiento de hardware;

- Factura por mantenimiento de hardware;

- Certificación presupuestaria para mantenimiento de hardware.

4.1 ¿Por qué aplicar el código de clasificación documental en el momento que se crea un documento?

De acuerdo Heredia (2010) "Será el código de clasificación, obtenido de dicho cuadro, para cada expediente, reconocido institucionalmente y utilizado como metadato por el 
Willian Fernando Ladino-Jiménez e María José Llanos Campoverde

sistema el que va a permitir a lo largo de la vida de los documentos -una vida que no termina en la selección- su identificación, su integridad, su inalterabilidad, su localización y su disponibilidad".

A raíz de las transferencias documentales realizadas desde las oficinas de gestión al Archivo Intermedio, se han podido detectar ciertas falencias en los documentos, tanto en su contenido y en su estructura formal.

- En la línea de referencia del documento a pesar de encontrarse en una misma oficina, contenían estructuras/denominaciones diferentes, en algunos casos se manejaba con acrónimos o por falta de espacio, se acortaba el nombre. Ejemplo:

$>$ Oficio Nº 384-06 UPS RECTORADO SEC. GENERAL

$>$ Oficio No. 384-06 UPS RECTORADO SECRETARIA GENERAL

$>$ Oficio Nº. 384-06 UPS SECRETARIA GENERAL

- Los documentos no contenían la línea de asunto, creando cierto tipo de incomodidad, debido a que ya sea una autoridad o colaborador tenía que leer todo el documento para conocer de qué se trataba.

¿Qué tiene que ver lo anterior con el CCD? Como se mencionó en el punto anterior, al considerarlo como un proceso de "sinapsis" deberán tener en cuenta los canales por los que serán trasmitidos los documentos o en el caso de los electrónicos, sus datos.

Al verificar el medio de transmisión de la información se detectó lo siguiente:

- El trámite se encuentra dividido, un porcentaje/una parte es enviado a través de mensajes de datos (e-mail), papel, sistemas informáticos propios desarrollados. Esto hace que el trámite se encuentre fragmentado en diferentes soportes;

- En soporte papel, duplicidad de documentos originales;

- En soporte electrónico: tipificación incorrecta, modificación de datos, impresión de los datos en soporte papel;

- Lugar de reposo del documento mientras finaliza el trámite;

- Generación de sentimiento de "dueño" de la información. 
En la propuesta se incluyó que además de consignar el código del CCD, al final del documento se pueda incluir el siguiente campo:

- Número de Trámite (NUT): este campo permite que cada documento perteneciente a un mismo trámite pueda estar encadenado bajo un mismo número. De manera mecanizada, obliga al usuario a tener en cuenta el número que le corresponde a un nuevo trámite o fijarse en el documento recibido el número que trae consigo para que lo pueda heredar.

\section{PROBLEMATICA ARCHIVISTICA}

Debemos reflexionar, a partir de los ejemplos puntuales expuestos, la creación y gestión de un Archivo Académico Universitario eficaz y eficiente en cuanto al cumplimiento de sus fines, no es un tema que se encuentre resuelto.

Existen por el contrario muchos problemas de diferente naturaleza, que van desde el puro sentido de "la propiedad de los papeles" por parte de las unidades administrativas gestoras, al de la ordenación y la conservación de los mismos una vez que han terminado su vida administrativa, pasando por la falta de normalización y de homogeneidad de los procesos administrativos a lo largo de los cuales se generan, a la falta de "respeto" a estos documentos, a la falta de técnicos formados y de aplicación de técnicas archivísticas básicas (como pueden ser las de los Cuadros de Clasificación Documental, de los Tiempos de Vida de cada Tipo Documental, de la simple eliminación de grapas, clips, alfileres e incluso de copias de documentos dentro de un expediente, de los expurgos, de las técnicas de conservación); de la selección, acondicionamiento y equipamiento de los espacios de Archivo e incluso, en el momento actual, de la "aparición" de sistemas de Administración Electrónica que han modificado en forma radical no solo la forma de elaborar y conservar los documentos, sino su propia naturaleza que deja de ser "material" para convertirse en "inmaterial, virtual o 
Willian Fernando Ladino-Jiménez e María José Llanos Campoverde

electrónica".

$\mathrm{Y}$ esto crea dos colecciones documentales en gran medida incompatibles, tanto en la gestión de su conservación y en las formas de acceso como en los sistemas, formatos y soportes de mantenimiento, sin hablar de la nueva naturaleza de los mecanismos de seguridad, tanto física como de autenticidad y autentificación. ¿Debemos hablar que, además de la humedad, temperatura, polvo, insectos y roedores de diferente naturaleza, debemos considerar la existencia de "hackers" cuyas intenciones y habilidades desconocemos?

\title{
IDENTIFICATION OF THE DOCUMENT ACCORDING TO THE CODE OF THE DOCUMENTARY CLASSIFICATION CHART: CASE UNIVERSIDAD POLITECNICA SALESIANA DEL ECUADOR
}

\begin{abstract}
SUMMARY
Due to the absence of a filing culture in an organization, the documents carry out an internal processing without having a control or knowing to which process it belongs, in the same way the terminology is unknown confusing "to conserve" as to incorporate the documents in a folder, envelope, and place them any physical space awaiting for the staff that works in a file. The "Universidad Politécnica Salesiana" in its organizational environment bets so that the document from its creation has consigned an identification according to the code of the documentary classification chart. The code will be reflected in the entire administrative and academic process accompanied of an alphanumeric structure that allows them to be grouped under the same number or subject procedure. The incorporation of the code and the alphanumeric structure will allow the staff to order the documentation according to the structure given in the Documentation Classification Chart.
\end{abstract}

Keywords: Qualification Chart. Documentary Types. File. Process. Classification Code. Document Management. 


\section{REFERENCIAS}

ARCHIVO GENERAL DE LA NACIÓN (Colombia). (1996). Gestión Documental: Bases para la elaboración de un programa.

HEREDIA HERRERA, A. (2010). Clasificación, cuadros de clasificación y e-gestión documental. Tábula, (13).

MILLÁN REYES, Ana Nieves; (2016). ROMERO RAMÍREZ, M. E. (Coord.) Conservación de documentos analógicos y digitales. San Sebastián: Nerea, 2015, 255 págs. ISBN: 978- 84-1625407-1. Anales de Documentación, 19(1).

MUNDET, J. R. C. (2001). Archivo y empresa: más allá de la historia. Transportes, Servicios y Telecomunicaciones, (1), 187-206 págs.

RODRÍGUEZ, A. D. (2010). La clasificación como proceso de gestión de documentos. Tábula, (13).

SÁNCHEZ GÓMEZ, A. E. (2007). Actualización de teorías archivísticas: una necesidad para mejorar la gestión de archivos venezolanos. Revista Venezolana de Información, tecnología y conocimiento.

Universitarios. . (2015). Curso Tratamiento Archivístico de Documentos y Gestión de Archivos UNIVERSIDAD POLITÉCNICA SALESIANA. Carta de Navegación 2014- 2018, pág.36. 